\title{
EVIDENCE FOR THE EXISTENCE OF NEUROTOXIC ESTERASE IN NEURAL AND LYMPHATIC TISSUE OF THE ADULT HEN*†
}

\author{
BenNiE Richard Dudek and Rudy J. Richardson $\neq$ \\ Toxicology Research Laboratory, Institute of Environmental and Industrial Health, School of Public \\ IIealth, The Univcrsity of Michigan, Ann Arbor, MI 48109, U.S.A.
}

(Received 12 January 1981; accepted 31 August 1981)

\begin{abstract}
Hen brain and spinal cord contain a number of esterases that hydrolyze phenyl valerate (PV). Most of this activity is sensitive to inhibition by micromolar concentrations of paraoxon. Included among the paraoxon-resistant esterases is neurotoxic estcrase (NTE), which is inhibited in vivo and in vitro by certain organophosphorus compounds, such as mipafox, which cause delayed neurotoxicity. Since published information on the NTE content of non-neural tissues was heretofore lacking, a comprehensive study was undertaken of the occurrence of this enzyme in tissues of the adult hen (Gallus gallus domesticus), the species of choice in the study of organophosphorus-induced delayed neurotoxicity. Complete differential titration curves of PV esterase activity were obtained by preincubation of each tissue homogenate with a wide range of concentrations of paraoxon, a non-neurotoxic compound, plus or minus mipafox, a neurotoxic compound, followed by PV esterase assay. Brain NTE activity was determined to be $2426 \pm 104 \mathrm{nmoles} \cdot \mathrm{min}^{-1}$. (g wet weight) ${ }^{-1}$ (mean \pm S.E.M.). Titration of other tissues resulted in the following NTE activities, expressed as percentages of brain NTE activity: spinal cord $(21 \%)$, peripheral nerve $(1.7 \%)$, gastrocnemius muscle $(0 \%)$, pectoralis muscle $(0 \%)$, heart $(14 \%)$, liver $(0 \%)$, kidney $(0 \%)$, spleen $(70 \%)$, spleen lymphocytes $(26 \%)$, and blood lymphocytes $(24 \%)$. Using an abbreviated procedure, erythrocytes and plasma showed no NTE activity. These results indicate that NTF has limited distribution among the tissues of the adult hen and is present in lymphatic as well as neural tissue.
\end{abstract}

Certain organophosphorus compounds cause a delayed degeneration of long axons in peripheral nerves and spinal cord of man, fowl, and certain other species [1,2]. This condition is referred to as delayed neurotoxicity. The hen was used in this study since this is the animal model commonly used in evaluation of delayed neurotoxicity potential of organophosphorus compounds [3]. In species sensitive to a single dose of neurotoxic compounds, there is a latent period of 8-14 days before clinical signs of ataxia and paralysis and histological evidence of distal-to-proximal "dying back" of affected axons appear. An early biochemical event in this neuropathy is the phosphorylation and aging of a particular phenyl valerate (PV) hydrolase in nerve tissue known as neurotoxic esterase (NTE) $\$$ [4-6]. Inhibition of hen brain NTE following dosing with neurotoxic organophosphorus compounds is highly correlated with the production of delayed neurotoxicity [7].

The NTE assay [5] is based on the differential sensitivity of PV esterases in hen brain homogenate

* This work formed a segment of the Ph.D. dissertation of B. R. D. and was presented in part at the Seventeenth Annual Meeting of the Society of Toxicology, San Francisco, CA, March 12-16, 1978.

$\dagger$ This research was supported in part by research grants R805339-01, USEPA, and ES01611-01, NIEHS, and by a faculty research grant from the Horace $H$. Rackham School of Graduate Studies, The University of Michigan.

$\ddagger$ Author to whom correspondence should be sent.

$\S$ A more precise name that we would like to suggest is neurotoxicant-sensitive esterase (NTSE). to neurotoxic and non-neurotoxic inhibitors. Preincubation at an appropriate concentration and time interval with paraoxon (diethyl $p$-nitrophenyl phosphate), a non-neurotoxic compound, inhibits about $80 \%$ of the total PV esterase activity. If mipafox $\left(N, N^{\prime}\right.$-diisopropyl phosphorodiamidofluoridate), a neurotoxic inhibitor, is included in the preincubation medium, an additional $10-17 \%$ of the activity is inhibited. This decrease in activity upon mipafox addition represents NTE activity.

This study was undertaken to provide criteria for identification of NTE activity in tissues of the hen in the form of complete inhibitor titration curves using the substrate phenyl valerate (PV) [8]. Previously, such curves have been presented for hen brain using the less sensitive substrate phenyl phenylacetate (PPA) [4] and, more recently, for human brain using PV [9]. It was of interest to rigorously determine whether this enzyme had exclusive localization in neural tissue in a given species or whether its distribution was more widespread.

\section{MATERIALS AND METHODS}

Animals. Spent White Leghorn hens, 1 to 1.5 years of age, weighing 1.5 to $2.0 \mathrm{~kg}$ were purchased from a local supplier. Hens were housed in stainless steel wire cages $0.8 \times 0.8 \times 0.9 \mathrm{~m}$ with $1.27 \mathrm{~cm}$ mesh stainless wire floors and were kept at least 2 weeks before use; birds were maintained in a temperature-controlled room on a diet of Purina Layena laying mash (Ralston Purina Co., St. Louis, MO) and tap water ad lib. A 12-hr light-dark cycle 
was maintained throughout the course of the experiment.

Chemicals. Paraoxon (diethyl $p$-nitrophenyl phosphate) was purchased from the Sigma Chemical Co. (St. Louis, MO) and was purified as described by Johnson [10]. Mass spectra of compound purified by this method were identical to published spectra [11]. PV and mipafox were synthesized and purified as described by Johnson [10]. Phenyl valerate was analyzed for impurities using i.r. spectroscopy and by assaying for phenol [12]. Both methods showed PV to be pure. Purity of mipafox was verified by melting point: found, $60-61^{\circ}$; published, $61-62^{\circ}[10]$. All other chemicals were analytical reagent grade or the highest grade commercially available; aqueous solutions were prepared using distilled-deionized water.

Tissue preparation. The buffer system used throughout for tissue homogenization and homogenate dilution was $50 \mathrm{mM}$ Tris- $\mathrm{HCl} / 2 \mathrm{mM}$ EDTA $\left(\mathrm{pH} 8.0,25^{\circ}\right)$. Tissues, except for lymphocytes, were kept at ice-bath temperature throughout processing, and were blotted before weighing. Homogenizations were generally done in 6.5 vol. of buffer in a glass-Teflon homogenizer with chamber clearance of $150-230 \mu \mathrm{m}$, using 15 strokes at $2300 \mathrm{rpm}$.

Since peripheral nerve and spinal cord required lengthy manipulation for removal, these tissues were removed from decapitated hens in a $4^{\circ}$ cold room. The peripheral nerve used was the endoneurial component of the ischiatic nerve; tissue was minced with scissors and homogenized using $30-35$ strokes in a glass-Teflon homogenizer and passed through a nylon screen $(333 \mu \mathrm{m})$ (TETKO, Inc., Elmsford, NY) stretched over the end of a truncated $10 \mathrm{ml}$ plastic syringe to remove residual connective tissue.

Gastrocnemius and pectoralis muscles were minced after removal of the thin collagenous sheaths, passed through a tissue press, homogenized, and passed through a nylon screen $(333 \mu \mathrm{m})$ to remove residual collagenous material.

Leucocytes from minced spleen supernatant fractions and from peripheral blood were isolated by centrifugation on Ficoll-Paque ${ }^{\mathrm{TM}}$ (Pharmacia, Piscataway, NJ) $[13,14]$. Weighed, pelleted cells were maintained at room temperature until just before use, at which time they were homogenized in buffer with a siliconized glass-Tcflon homogenizer (chamber clearance of $100-150 \mu \mathrm{m}$ ). Cells were quantified on the basis of wet weight. Cells obtained by this method were checked for identity and purity using light (Wright's stain) [15] and electron microscopy. This preparation yielded $\geq 95 \%$ lymphocytes.

Differential titrations. The NTE assay was done essentially according to the method described previously [5]. Complete paraoxon titration curves were generated by varying the paraoxon concentration in the preincubation medium, over the concentration range 0.01 to $1000 \mu \mathrm{M}$. For tissues that showed very high PV esterase activity at low paraoxon concentrations, it was necessary to use an aliquot of the preincubation mixture for incubation with PV. For each tissue studied, an inspection of the paraoxon titration curve, together with consideration of the

\footnotetext{
* Panel B of Fig. 1 is from Ref. 3.
}

absolute activity remaining at the higher paraoxon concentrations, yielded an optimum paraoxon concentration, usually $10-100 \mu \mathrm{M}$, for use in the mipafox titration.

The mipafox titration curves were generated by including, in the preincubation medium, paraoxon at the selected optimum concentration, plus mipafox, dissolved in Tris-EDTA buffer, over the concentration range 0.001 to $1024 \mu \mathrm{M}$.

In the cases of lymphocytes and peripheral nerve, assay volumes were reduced by factors of 20 and 2 , respectively, to accommodate smaller amounts of tissue.

For each tissue studied, at least three complete titration curves were obtained using each inhibitor. Data are presented as means \pm S.E.M.

\section{RESULTS}

Panels A and B* of Fig. 1 are the neurotoxic and non-neurotoxic inhibitor titration curves for hen brain PV esterase activity. They serve to illustrate
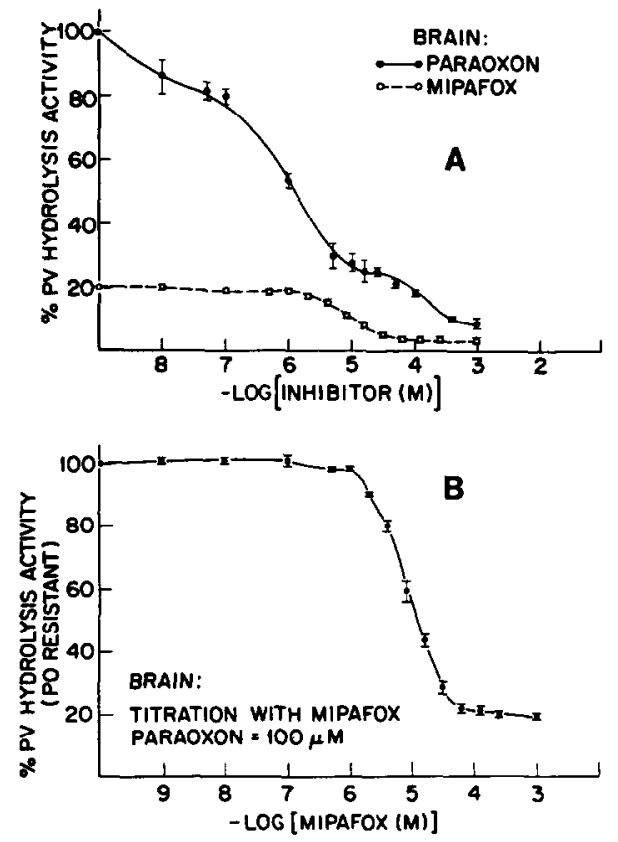

Fig. 1. (A) Effects of increasing concentrations of paraoxon alone (O) and of increasing concentrations of mipafox in the presence of paraoxon $(O)$ on the hydrolysis of phenyl valerate (PV) by hen brain homogenate. The concentration of paraoxon used in the mipafox titration is indicated on curve B. (B) Effect of mipafox on the paraoxon-insensitive hydrolysis of phenyl valerate by hen brain homogenate; $100 \%=$ activity remaining that is insensitive to paraoxon before addition of mipafox. The concentration of paraoxon used in the mipafox titration is indicated on the graph. Tissue homogenates were preincubated for $20 \mathrm{~min}$ with inhibitors before addition of substrate, whereupon incubation was continued for an additional $15 \mathrm{~min}$ before stopping the reaction and analyzing for phenol produced as described in Materials and Methods. Each point is the mean \pm S.E.M. from at least three separate titration experiments. [Reproduced by permission from C. S. Davis and R. J. Richardson, in Experimental and Clinical Neurotoxicology (Eds. P. S. Spencer and H. H. Schaumburg), p. 538. Williams \& Wilkins, Baltimore (1980).] 


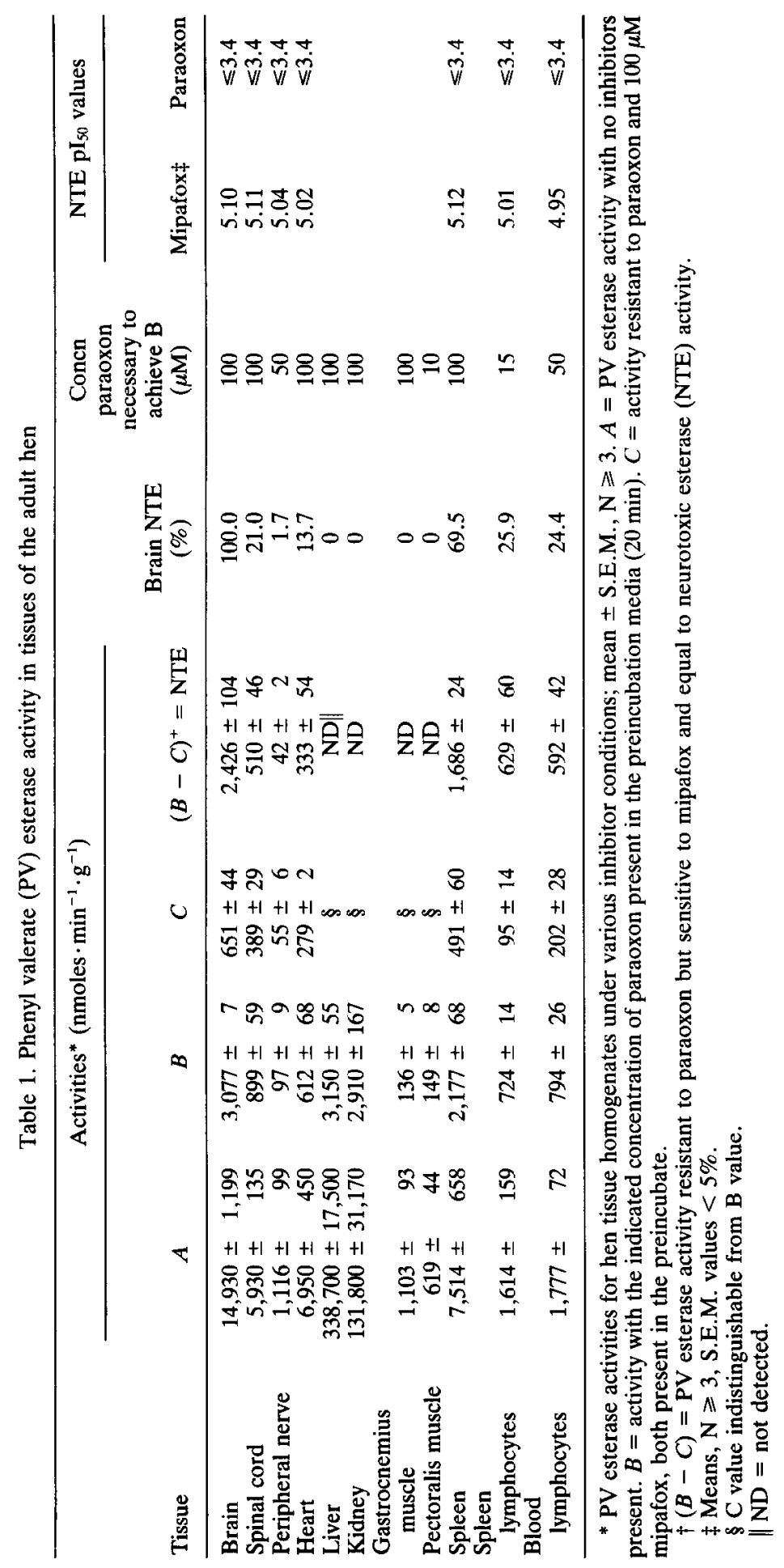


the experimental design and define the criteria used for identification and quantitation of NTE in brain and other tissues. The curves result from the differential sensitivities of brain PV esterases to nonneurotoxic paraoxon and to neurotoxic mipafox. The $100 \%$ value on Fig. $1 \mathrm{~A}$ corresponds to the total brain PV esterase activity referred to as activity $A$ (see Table 1). Activity $A$ for hen was 14,930 nmoles PV hydrolyzed $\cdot \min ^{-1} \cdot(\mathrm{g} \text { wet weight tissue })^{-1}$. Approximately $70-75 \%$ of this activity was sensitive to $10-100 \mu \mathrm{M}$ paraoxon. The level of PV esterase activity resistant to a paraoxon concentration of $100 \mu \mathrm{M}$ was activity $B$ (see Table 1 ). Activity $B$ for hen brain was 3077 nmoles PV hydrolyzed $\cdot \mathrm{min}^{-1} \cdot(\mathrm{g}$ wet weight tissue) $)^{-1}$.

The lower curve in Fig. 1A represents the titration of activity $B$ with the neurotoxic inhibitor mipafox. This curve is shown on an expanded scale in Fig. 1B. The PV esterase activity resistant to both paraoxon and mipafox is referred to as activity $C$ or inhibitor-resistant esterase activity (IRE). The difference $(B-C)$ is the portion of the paraoxon-resistant PV esterase activity that is also sensitive to mipafox; this is the NTE activity which for brain equalled $2426 \mathrm{nmoles} \mathrm{PV}$ hydrolyzed $\cdot \mathrm{min}^{-1} \cdot(\mathrm{g}$ wet weight tissue $)^{-1}$. The mipafox concentration corresponding to $C$ was $100 \mu \mathrm{M}$. Analysis of the brain curves revealed that the $(B-C)$ activity represented PV esterase activity with $\mathrm{pI}_{50}$ values for paraoxon of $\geq 3.4$ and for mipafox 5.1. These values established a set of criteria for NTE identification in the other tissues studied. Similar titrations were carried out on other tissues and analyzed in like manner to brain [8]. The results are summarized in Table 1. Plasma and erythrocytes contained little, or no, total PV esterase activity, and so complete titrations were not performed. Any activity present was greatly reduced by low ( $\leqslant 10 \mu \mathrm{M})$ concentrations of paraoxon, with no additional effect produced by mipafox. Therefore, NTE activity was concluded to be nil in erythrocytes and plasma. NTE activity was confirmed for spinal cord and peripheral nerve, as reported earlier using the substrate PPA [4] and by single inhibitor concentration using PV [5]. In spite of high total PV esterase activity in liver and kidney, most was sensitive to paraoxon and no differential sensitivity to mipafox could be detected. Likewise, gastrocnemius and pectoralis muscles showed no differential sensitivity by this type of analysis. Heart did exhibit a differential sensitivity equal to $13.7 \%$ of brain NTE activity on a wet weight basis. However, the shape of the mipafox titration curve for heart was dissimilar to that obtained for brain. The most striking results were those obtained for spleen, spleen lymphocytes, and peripheral blood lymphocytes. The mipafox titration curves all bore a striking qualitative resemblance to the brain curve and yielded $\mathrm{pI}_{50}$ values within $5 \%$ of those of brain NTE.

\section{DISCUSSION}

The data presented in this study reconfirm the presence of NTE in the nervous system and for the

* B. R. Dudek and N. Mottershaw-Jackson, unpublished data. first time demonstrate the presence of NTE in heart and lymphoid tissue of the adult hen.

Concerning the other tissues studied, it should be pointed out that the muscles used in this work - the gastrocnemius and the pectoralis-are both composed of a large proportion of fast fibers[16]. Innervation density in muscles, i.e. the number of motor end plates per fiber, is known to be inversely related to the speed of the fiber type present [17]. This distinction, relating to the quantity of end plates per fiber, is important, because there is evidence indicating that NTE is present in synaptosomes isolated from hen brain [18]. If the motor end plate is considered a peripheral counterpart of the synaptosome and since the muscles studied had a low quantity of end plates, NTE activity present in these muscles may have been at levels below the detection limits of this method. Using the anterior latissimus dorsi muscle of the hen, which is composed exclusively of slow fibers [19], we have detected NTE activity equal to $75.8 \pm 16 \mathrm{nmoles} \cdot \mathrm{min}^{-1} \cdot(\mathrm{g} \text { wet weight tissue })^{-1}$ (mean \pm S.E.M., $\mathrm{N}=3$ ).*

Heart muscle is richly innervated by the autonomic nervous system and is generally compared with slow rather than fast skeletal muscle [20]. It is possible that the synaptic junctions associated with this muscle are the source of NTE activity in hen heart, but this point will require further investigation in order to be resolved.

The presence of NTE activity in hen lymphocytes, as evidenced by similarities to brain in response to inhibitors during differential titration of lymphocyte phenyl valerate esterase activity, creates important avenues for further investigation. Because inhibition of hen brain and spinal cord NTE is closely associated with the production of delayed degeneration of axons by neurotoxic organophosphorus compounds [7], NTE inhibition in lymphocytes may, in turn, be correlated with inhibition of NTE in brain and other neural tissue. Such a correlation would make possible the sampling of peripheral blood components to mcasure cxposure to ncurotoxic organophosphorus compounds and to assess concomitant neurotoxic hazard. Lotti and Johnson [21] have shown recently that hen blood lymphocyte NTE is not a correlatc of brain NTE during chronic exposure to neurotoxic compounds. However, after acute exposure ( $4 \mathrm{hr}$ ), hen blood lymphocytc NTE inhibition was highly correlated with hen brain NTE inhibition [8]. Additionally, the existence of NTE in lymphocytes may facilitatc its biochemical study by providing a source of the enzyme from a homogeneous population of relatively simple cells. A distinct advantage of using these cells in lieu of a more complcx neural system for isolation or subcellular localization is that intra- and intercellular cross-contamination in subccllular fractions can be avoided. Synaptosomes prepared from brain, for example, are known to be heavily contaminated with glial cell membranes [22] and brain microsomal fractions contain membranes derived from the endoplasmic reticulum and plasma membranes of both neurons and glia [18]. On the other hand, relatively pure preparations of, for example, plasma membranes may be prepared from lymphocytes by several techniques [23,24]. Cultured lymphocytes could potentially be used as a source 
of NTE for study or for screening compounds for delayed neurotoxicity potential. Such a system could be used to study biochemical or functional effects of NTE phosphorylation in a population of cells that is simpler and better defined than a neural system. Leukocytes have been employed in a number of studies as possible models of neural function, especially with respect to membrane changes brought about by interaction with drugs, hormones, or neurotransmitters $[25,26]$.

Finally, it has been suggested that the pathogenesis of organophosphorus-induced delayed neurotoxicity may have an immunological basis [27,28]. Even though this contention has not found experimental support [29], the finding of NTE in lymphatic as well as neural tissue is intriguing and points to a need to further investigate the neuroimmunological aspects of this problem.

\section{REFERENCES}

1. J. B. Cavanagh, J. Neurol. Neurosurg. Psychiat. 17, 163 (1954).

2. J. B. Cavanagh, Int. Rev. expl Pathol. 3, 219 (1964).

3. C. S. Davis and R. J. Richardson, in Experimental and Clinical Neurotoxicology (Eds. P. S. Spencer and H. H. Schaumburg), pp. 527-44. Williams \& Wilkins, Baltimore (1980).

4. M. K. Johnson, Biochem. J. 114, 711 (1969).

5. M. K. Johnson, CRC Crit. Rev. Toxic. 3, 289 (1975).

6. M. K. Johnson, J. Neurochem. 23, 785 (1974).

7. M. K. Johnson, Archs Toxic. 34, 259 (1975).

8. B. R. Dudek, Ph.D. Thesis, The University of Michigan, Ann Arbor, MI (1979).

9. M. Lotti and M. K. Johnson, J. Neurochem. 34, 747 (1980).
10. M. K. Johnson, Archs Toxic. 37, 113 (1977).

11. J. N. Damico, J. Ass. off. analyt. Chem. 49, 1027 (1966).

12. R. N. Martin, Analyt. Chem. 21, 1419 (1949).

13. A. Böyum, Scand. J. clin. Lab. Invest. 21 (Suppl. 97), 9 (1968).

14. Anonymous, Ficoll-Paque: for In Vitro Isolation of Lymphocytes. Pharmacia Fine Chemicals, Piscataway, NJ (1975).

15. A. M. Lucas and C. Jamroz, Atlas of Avian Hematology, U.S. Department of Agriculture, Washington, DC (1961).

16. J. M. Wilkinson, Biochem. J. 169, 229 (1978).

17. A. J. Hess, J. Physiol., Lond. 157, 221 (1961).

18. R. J. Richardson, C. S. Davis and M. K. Johnson, J. Neurochem. 32, 607 (1979).

19. G. F. Gauthier, S. Lowey and A. W. Hobbs, Nature, Lond. 274, 25 (1978).

20. S. Sartore, S. Pierobon-Bormioli and S. Schiaffino, Nature, Lond. 274, 82 (1978).

21. M. Lotti and M. K. Johnson, Archs Toxic. 45, 263 (1980).

22. W. Sieghart, A. Sellstrom and F. Henn, J. Neurochem. 30, 1587 (1978).

23. J. W. DePierre and M. L. Karnovsky, J. Cell Biol. 56, 275 (1973).

24. D. I. Kalish, C. M. Cohen, B. S. Jacobson and D. Branton, Biochim. biophys. Acta 506, 97 (1978).

25. F. Medzihradsky, M. J. Marks and J. I. Metcalfe, $A d v$. biochem. Psychopharmac. 8, 537 (1974).

26. A. Lopker, L. G. Abood, W. Hoss and F. J. Lionetti, Biochem. Pharmac. 29, 1361 (1980).

27. R. D. O'Brien, Insecticides: Action and Metabolism, p. 59. Academic Press, New York (1967).

28. P. G. Watanabe and R. P. Sharma, J. Toxic. environ. Hlth 1, 777 (1976).

29. P. G. Watanabe and R. P. Sharma, Archs environ. Contam. Toxic. 6, 233 (1977). 